\title{
Tibor Cserháti: Chromatography of aroma compounds and fragrances
}

\author{
Bogusław Buszewski
}

Published online: 18 August 2010

(C) Springer-Verlag 2010

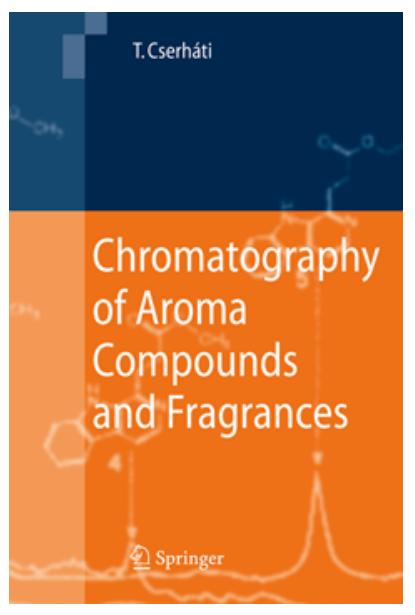

Bibliography

Chromatography of aroma compounds and fragrances

Tibor Cserháti

Springer

ISBN: 978-3-642-01655-4

Hardcover, 392 pages,

January $2010,159,95 €$

Book's topic The chemical composition of the aroma of foods and various food products is related to the occurrence of flavour compounds. The quantity and existence of flavour compounds in food products affect consumer acceptance. Modern analytical methods enable analysis of aroma compounds belonging to different groups.

This book covers a wide range of applications of analytical techniques, particularly gas chromatography coupled with mass spectrometry, in the analysis of flavour and fragrance compounds. Many examples of analyses of food, food products, fruit, vegetables, beverages, spices and

\footnotetext{
B. Buszewski $(\square)$

Dept. of Environmental Chemistry \& Bioanalytics,

Faculty of Chemistry, Nicolaus Copernicus University,

7 Gagarin St.,

87100 Toruń, Poland

e-mail: bbusz@chem.uni.torun.pl
}

other products, including essential oils have been described. Besides description of the composition of food and food products, two chapters also cover the biological effects of flavour compounds and aspects of environmental pollution (air, water, soil, sediment, sewage, and others).

Aroma substances have been designated as biologically active compounds. Many of these compounds have beneficial biological activity and can be used in human health care as ingredients of various medicaments. However, some aroma compounds are allergens and artefacts, and it is important to eliminate these during the preparation of natural medicaments. For that reason method validation by confirmation of identity and determination of selectivity, specificity, LOD, LOQ, linearity, repeatability, precision, accuracy, and uncertainty is needed.

Contents The book consists of five chapters. Abbreviations and an index are supplements to the book. References have been added to the end of each chapter. The first chapter deals with the theory and practice of chromatographic techniques. Sample-preparation methods, in particular preconcentration and prepurification, have been taken into consideration. Principles of GC, LC, including TLC and HPLC, and electrically driven chromatography have been presented.

The next two chapters cover chromatographic results from analysis of food, food products, and essential oils. Examples from analysis of fruit, legumes, other vegetables, cereals products, edible oils, meat, meat products, milk, dairy products, non-alcoholic and alcoholic beverages, coffee, tea, cocoa, spices, other food products, and essential oils have been presented. Also, results from application of enrichment techniques, the use of chromatography, includ- 
ing GC coupled with MS, multi-dimensional GC-MS, TLC, HPLC, gel electrophoresis, etc., have been given. Many analytical conditions have been described in detail. Specific results from quantitative analysis of many flavour compounds in real food and food products have been included. Besides results obtained from analysis of flavour compounds, examples of analysis of peptide profiles and casein degradation products in cheese by RP-HPLC and urea-polyacrylamide gel chromatography have been presented. Moreover, applications of other techniques have been given, for example the culture-independent fingerprint technique, PCR, and denaturing gradient gel electrophoresis (DGGE), which have been used for investigation of microbial dynamics during the manufacture of one kind of cheese.

Chapter 4 covers biochemical and biophysical aspects of the formation of natural aroma compounds. The biosynthetic process and the enzymatic procedure resulting in the production of volatile organic compounds have also been described. Artefact formation during extraction procedures, the sorption of aroma compounds on complex matrices, the determination of various emission patterns, and, finally, toxicity studies of flavours have also been taken into consideration.

The last chapter (Chapter 5) concerns the problem of the environmental pollution. Many natural and synthetic fragrances can occur in a variety of environmental matrices and, through this medium, affect the human body and wildlife-it is commonly known that some pollutants, including synthetic fragrances, are persistent, liable to bioaccumulation, and have toxic properties. Because of all these properties, it is necessary to promptly and rapidly develop procedures for separation and analysis of pollutants in environmental samples. In Chapter 5 many results from determination of pollutants in ground, surface, and waste water, sludge, and miscellaneous environmental matrices have been presented.

Comparison with existing literature This book is a useful supplement to other well-established publications in the field of application of chromatographic techniques to the analysis of aroma compounds and fragrances. The literature cited is appropriately selected for the material presented. The book is a good review of current knowledge about qualitative and quantitative analysis of food, food products, fruit, vegetables, beverages, spices, essential oils, etc., and environmental samples.

Readership recommendation This book is a compendium of achievements in the separation and determination of aroma compounds and fragrances. Topics presented in the book could be of interest to scientists working in the field of flavours and fragrances, analytical chemists, employers in the food industry, research workers and development groups, PhD students, among others.

Summary Chromatography of Aroma Compounds and Fragrances is a well-written publication and a rich source of information. The presented principles of chromatographic techniques and results achieved by international research groups around the world are very useful not only for researchers active in this field, but also students, etc. 